\title{
Practical Implementation of Compressive Sensing for High Resolution STEM
}

Dorothea Mücke-Herzberg ${ }^{1}$, Patricia Abellan ${ }^{1}$, Michael C. Sarahan ${ }^{1, *}$, Iain S. Godfrey ${ }^{1}$, Zineb Saghi ${ }^{2}$, Rowan K. Leary ${ }^{2}$, Andrew Stevens ${ }^{3,4}$, Jackie Ma ${ }^{5}$, Gitta Kutyniok ${ }^{5}$, Feridoon Azough ${ }^{6}$, Robert Freer ${ }^{6}$, Paul A. Midgley ${ }^{2}$, Nigel D. Browning ${ }^{4}$, Quentin M. Ramasse ${ }^{1}$

1. SuperSTEM Laboratory, SciTech Daresbury Campus, Daresbury, U.K.

2. Department of Materials Science and Metallurgy, University of Cambridge, Cambridge, U.K.

3. Dept. of Elec. and Comp. Engineering, Duke University, Durham, USA

4. Pacific Northwest National Laboratory, Richland, USA

5. Technische Universität Berlin, Berlin, Germany

${ }^{6}$ School of Materials, Materials Science Centre, University of Manchester, Manchester M13 9PL, U.K.

*. Now at Continuum Analytics, Austin, Texas, USA

New generation scanning transmission electron microscopes (STEMs) allow for atomic resolution imaging and spectroscopy at low beam energy, in many cases below the threshold for primary knock-on damage. For many samples, however, including many biological specimens, lowering the acceleration voltage does not prevent damage as radiolysis is the dominant mechanism. Although a number of lowdose high resolution (HR) schemes have been explored with some level of success [1], the promise held by so-called compressive sensing (CS) approaches is yet to be fully exploited [2].

Typically, the Nyquist frequency determines the sampling rate required to resolve a specific feature in a dataset and as such provides a lower limit for the electron dose necessary to acquire the data experimentally. In practice this requires oversampling by twice the maximum relevant frequency. However, by applying the CS principles of sparsity and incoherence this limit can be overcome. CS theory then allows to recover the essential image information from randomly subsampled images. The theory of CS has been successfully applied in a number of areas, e.g. astronomy, MRI scanning and electron tomography [3]. As STEM, both imaging [4] and spectroscopy, is a controllable scanning probe technique it lends itself to the CS approach. Despite this huge potential, CS acquisition schemes able to recover quantitative information have so far not been implemented for scanning transmission electron microscopy.

Here we demonstrate such a CS-based STEM acquisition implementation and benchmark its performance at atomic-resolution using a variety of systems, including as illustrated in fig. 1, complex oxide ceramics with an inhomogeneous distribution of point defects, but also highly beam-sensitive materials for catalysis with atomic resolution [5]. The implementation relies on custom-built hardware and software which control an electrostatic beam shutter to blank the electron beam during all but a few randomly chosen pixels through a regular image (or indeed spectrum image) acquisition. The total electron dose used to form a whole dataset can thus be drastically reduced whilst maintaining all other acquisition parameters identical to regular data acquisition, allowing a seamless transition from 'fully sampled' to ultra-low-dose conditions. We show that datasets acquired in this fashion down to a few $\%$ of the total incoming dose can be reconstructed to yield a truthful atomic resolution representation of the sample, using a variety of reconstruction algorithms $[4,6]$. We show that this implementation of CS can also be extended to 2D spectroscopy mapping and simultaneous HAADF image acquisition, see fig. 2. These results clearly open the door to practical ultra-low dose high-resolution imaging and spectroscopy in the STEM for very beam sensitive samples, where the level of sampling can simply be chosen depending on the damage threshold of the material being investigated [7]. 
References:

[1] J.P. Buban et al., J. Electron. Microsc. 59 (2010), 103-112

[2] P. Binev et al., Compressed Sensing and Electron Microscopy, in Modeling Nanoscale Imaging in Electron Microscopy, T. Vogt et al., Eds., Springer (2012)

[3] Z. Saghi et al., Nano Letters 11 (2011), 4666

[4] A. Stevens et al., Microscopy 63 (2014), 41-51

[5] D. Mücke-Herzberg et al. submitted

[6] J. Ma. et al. submitted

[7] SuperSTEM is the UK EPSRC National Facility for Aberration-Corrected STEM, supported by the Engineering and Physical Science Research Council. PNNL, a multiprogram national laboratory, is operated by Battelle for the U.S. Department of Energy under Contract No. DE-AC05-76RLO1830.

A)
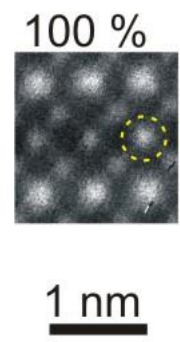
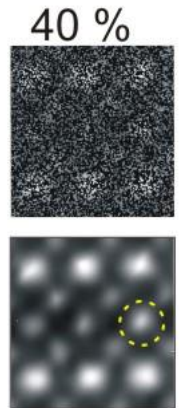

Reconstructed Images
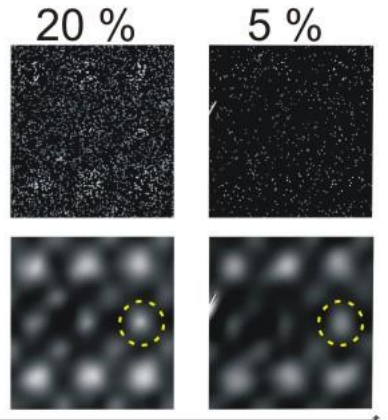

B)
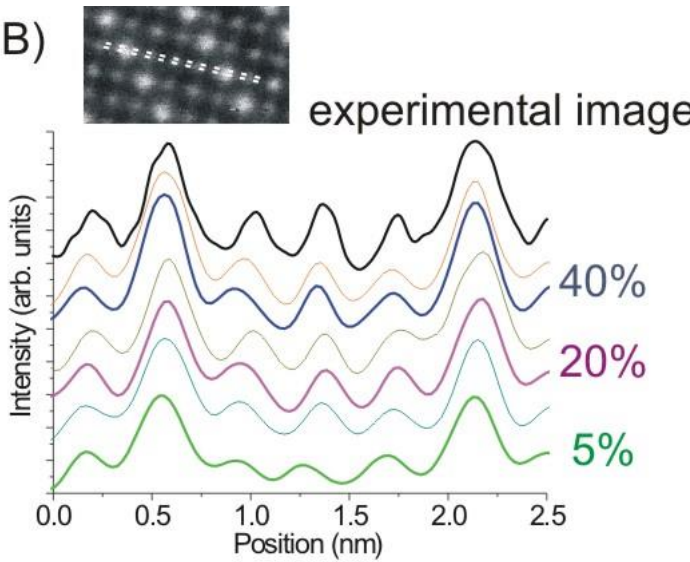

Figure 1. Example CS shearlet reconstruction (see [6]) of NTCT at different values of undersampling showing correct identification of point defects on the left and comparative intensity profiles for different values of undersampling on the right [5].

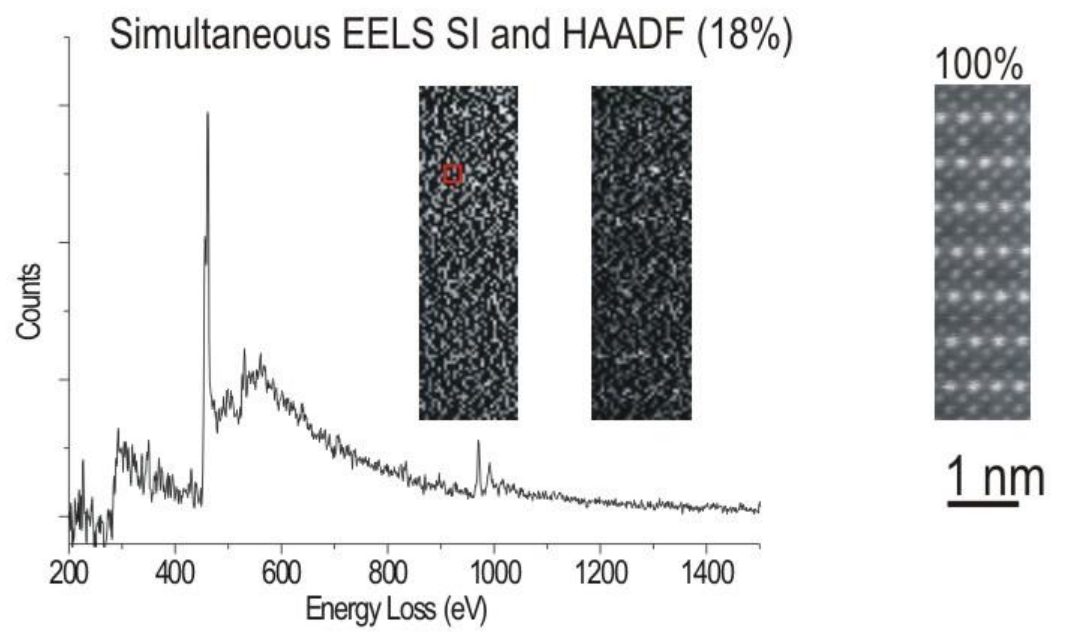

Figure 2. Example CS EELS acquisition [5]. 